\title{
Far-Infrared Cyclotron Resonance Study of Electron Dynamics in (BEDT-TTF) ${ }_{2} \mathrm{KHg}(\mathrm{SCN})_{4}$
}

\author{
J. Singleton, (1) F. L. Pratt, ${ }^{(1)}$ M. Doporto, ${ }^{(1)}$ T. J. B. M. Janssen, ${ }^{(2)}$ M. Kurmoo, ${ }^{(3)}$ \\ J. A. A. J. Perenboom, ${ }^{(2)}$ W. Hayes, ${ }^{(1)}$ and P. Day ${ }^{(3)}$ \\ (1) Department of Physics, The Clarendon Laboratory, University of Oxford, Parks Road, Oxford OXI 3PU, United Kingdom \\ ${ }^{(2)}$ High Field Magnet Laboratory, University of Nijmegen, Toernooiveld I, NL-6525 ED Nijmegen. The Netherlands \\ ${ }^{(3)}$ Royal Institution, 21 Albermarle Street, London WIX $4 B S$ \\ and Inorganic Chemistry Laboratory, University of Oxford, South Parks Road, Oxford OXI 3QR, United Kingdom
}

(Received 30 September 1991)

\begin{abstract}
We report the first measurement of cyclotron resonance in a metallic charge-transfer salt. Carrier pockets are observed with effective masses of $0.40 m_{e}$ and $0.94 m_{e}$. In contrast, masses of $2.0 m_{e}$ and $2.4 m_{e}$ are seen in magnetotransport. We propose that the transport masses are enhanced by electronelectron interactions, whereas, following Kohn's theorem, the mass measured by cyclotron resonance is independent of these interactions. These measurements therefore represent the first direct gauge of the electron-electron interaction in a metallic charge-transfer salt.
\end{abstract}

PACS numbers: $71.20 . \mathrm{Hk}, 71.25 . \mathrm{Hc}, 76.40 .+\mathrm{b}$

The properties of charge-transfer salts of the molecule BEDT-TTF [1] encompass many of the disciplines of condensed matter physics. One member of this family of two-dimensional materials has provided us with the highest-temperature organic superconductor; yet another combines antiferromagnetism with metallic conductivity. By varying the anion $X$ incorporated into the salt (BEDT-TTF $)_{n} X$, the stoichiometry and band filling are modified, producing compounds which are metallic, semimetallic, or semiconducting [2]. The compounds are also of interest in the study of electron-electron interactions (EEI) in narrow-bandwidth metallic systems; a large enhancement of the transport effective mass over the predicted band mass in metallic (BEDT-TTF) ${ }_{2}-$ $\mathrm{Cu}(\mathrm{SCN})_{2}$ has been noted and associated with EEI $[3,4]$.

Experimental techniques which gauge the Fermi surface are making a great contribution to the understanding of these materials. Most studies have involved measurements of the Shubnikov-de Haas oscillations (SdHO) in the magnetoresistance (MR) [5]; there have also been a few de Haas-van Alphen studies [6]. In contrast, no cyclotron resonance (CR) studies have been reported (although an apparent resonance has recently been observed in the field-induced spin-density-wave state of TMTSF $_{2-}$ $\mathrm{ClO}_{4}$ [7]). $\mathrm{CR}$ measurements have an important role to play in such studies since, according to Kohn's theorem [8], the effective mass measured by CR is independent of EEI, giving the band mass renormalized only by electron-phonon interactions, whereas the transport mass, measured, e.g., by SdHO, will additionally be influenced by the EEI. In this paper we report the first far-infrared (FIR) optical measurements of the cyclotron mass of the closed sections of the Fermi surface in metallic (BEDT$\mathrm{TTF})_{2} \mathrm{KHg}(\mathrm{SCN})_{4}$. In addition, we have measured the mass renormalized by EEI using SdHO. This is found to be several times larger than the cyclotron mass, providing clear evidence of the importance of EEI in this material.

Measurements were performed on crystals of $(\mathrm{BEDT}-\mathrm{TTF})_{2} \mathrm{KHg}(\mathrm{SCN})_{4}$ grown electrochemically us- ing BEDT-TTF prepared by the method of Larsen and Lenoir [9] with $\mathrm{KSCN}, \mathrm{HgSCN}$, and 18Crown6 in a mixture of $90 \% 1,1,2-\mathrm{TCE}$ and $10 \%$ ethanol. Crystals formed as small black distorted-diamond-shaped platelets. This salt has the typical layer structure for a BEDT-TTF salt, where highly conducting BEDT-TTF layers are separated by layers of anions [10-14].

$(\mathrm{BEDT}-\mathrm{TTF})_{2} \mathrm{KHg}(\mathrm{SCN})_{4}$ is metallic down to at least $400 \mathrm{mK}$ and is known to be in an antiferromagnetic ground state below $\sim 10 \mathrm{~K}[10]$. The salt has attracted much attention recently due to the spin-split structure of the SdHO and the anomalous high-field "kink" at $\sim 23 \mathrm{~T}$ in the MR seen in early studies [10-13]; our MR measurements on this material [14] have shown a number of new features. Above the kink at $23 \mathrm{~T}$, the SdHO, which show only one frequency (plus harmonics) at $656 \pm 10 \mathrm{~T}$, and the angle-dependent MR are both in fair agreement with the theoretical Fermi surface predicted using the extended Hückel model [15], which has only one rather isotropic closed orbit (see inset to Fig. 1). Figure 1 shows the oscillatory component of the MR up to $20 \mathrm{~T}$ at $0.5 \mathrm{~K}$, and illustrates the rather different behavior which occurs below the kink feature: Besides the primary series of $\mathrm{SdHO}$ with a frequency of $\sim 670 \pm 5 \mathrm{~T}$, believed, like the single frequency observed above the kink, to be associated with the $V$-point hole pocket, there is an extra weaker series with a sample-dependent higher frequency which can be as large as $\sim 860 \mathrm{~T}$ [14]; the beating between the two frequencies can be clearly discerned in Fig. 1. The extra frequency is thought to be due to an extra closed section of Fermi surface resulting from the effect of the interplane antiferromagnetic ordering on the band structure. The kink feature may therefore represent a fieldinduced change in the interplane ordering [14].

At fields below the kink feature, we are thus dealing with two closed 2D pockets of carriers. In order to measure the transport masses for these carrier pockets, $m_{\mathrm{trl}}^{*}$ and $m_{\mathrm{tr} 2}^{*}$, MR measurements were performed at fields well below the kink transition at both ${ }^{3} \mathrm{He}$ and ${ }^{4} \mathrm{He}$ tem- 


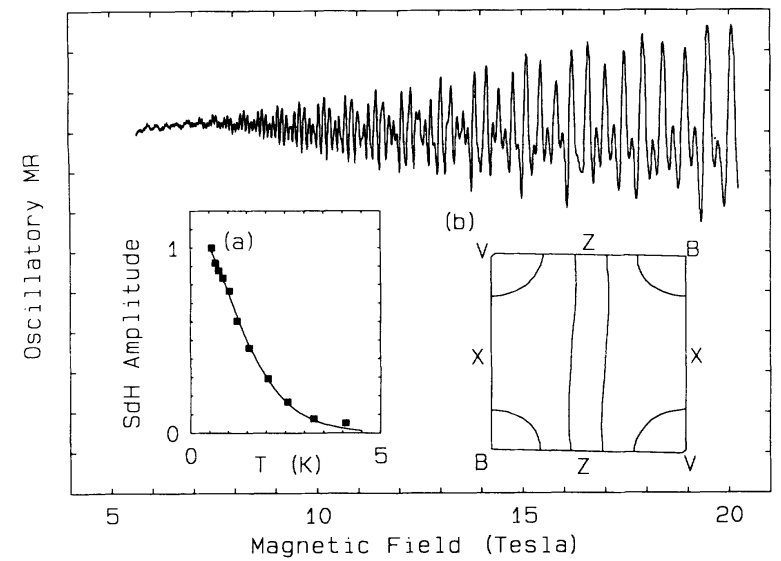

FIG. 1. The oscillatory component of the MR of a crystal of (BEDT-TTF) ${ }_{2} \mathrm{KHg}(\mathrm{SCN})_{4}$ at $0.5 \mathrm{~K}$ (from Ref. [14]), showing beats between the two series of SdHO. Inset (a): The measured temperature dependence of the Fourier amplitude of the primary 670-T oscillation series (solid squares) fitted using $m^{*} / m_{i}=1.98$ (line). Inset (b): The closed pocket in the calculated Fermi surface [15], which we associate with the primary CR.

peratures; temperatures were measured using calibrated $\mathrm{RuO}$ and $\mathrm{Ge}$ resistance thermometers. The temperature dependence of the amplitude of the primary 670-T SdHO between 17 and $20 \mathrm{~T}$ is shown as an inset to Fig. 1; it is consistent with an effective mass $m_{\mathrm{trl}}^{*}=2.0 m_{e}$, similar to but somewhat higher than the previously reported value of $1.4 m_{e}$ [13], which was measured over a more restricted temperature range. The SdHO of the secondary, higherfrequency series are rather weaker, and it is not possible to make a meaningful estimate of the effective mass using the same method. Instead, we have performed harmonic ratio experiments [16] on both series, in which the ratio of the fundamental frequency of the SdHO to its second harmonic is measured as a function of sample orientation (see [17] for a review of the technique). These indicate that the ratio of the masses is $m_{1 \mathrm{r} 1}^{*} / m_{\mathrm{tr} 2}^{*} \simeq 1.22$, so that $m_{\text {tr } 2}^{*}=2.4 m_{e}$.

For the CR measurements the crystals were arranged in the form of a mosaic with the highly conducting planes perpendicular to the magnetic field. The FIR laser system used has been described in detail elsewhere [18]; two carbon bolometers were used to measure the incident and the reflected radiation, and the reflected signal was normalized to the incident signal. Magnetic fields were provided by a Bitter magnet.

The measured FIR magnetoreflectivity at $1.2 \mathrm{~K}$ is shown in Fig. 2 for several different laser wavelengths. Two sharp features are observed, occurring at fields which are proportional to the FIR energy, the characteristic behavior of $\mathrm{CR}$; it should be noted that we have independently measured electron-spin resonance (ESR) corresponding to $g^{*}=2$ in (BEDT-TTF) ${ }_{2} \mathrm{KHg}(\mathrm{SCN})_{4}$, and that neither of the observed resonances is narrow

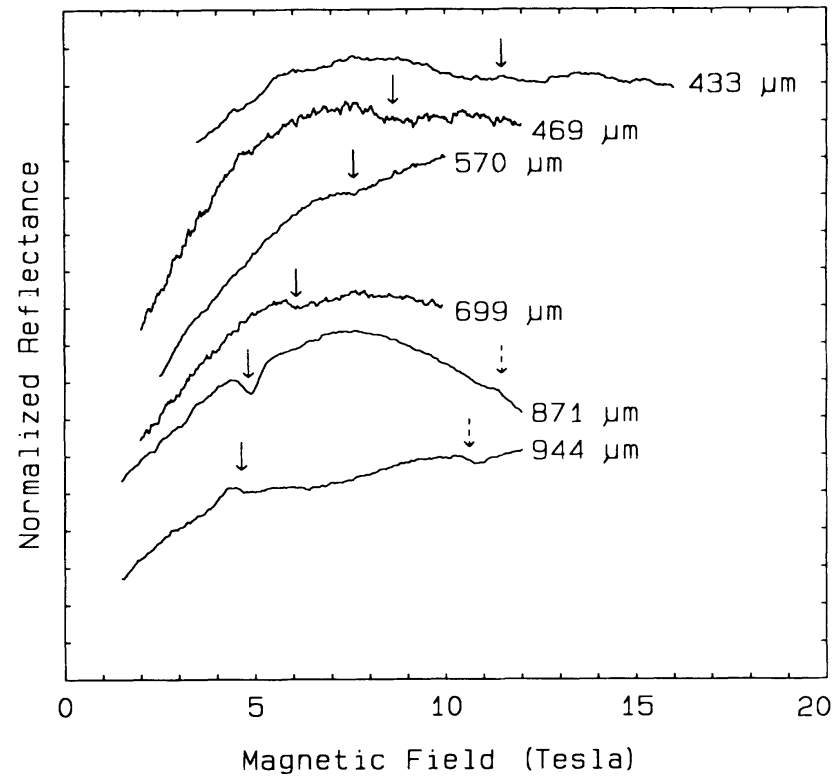

FIG. 2. Normalized magnetoreflectance of (BEDTTTF ${ }_{2} \mathrm{KHg}(\mathrm{SCN})_{4}$ at $1.2 \mathrm{~K}$ for different radiation energies. The CR features are marked with arrows.

enough, nor at a high enough field, to be ESR. The resonances are superimposed on the gently varying background magnetoreflectance and instrumental response. The strongest of the two features appears as a dip in reflectivity at $5 \mathrm{~T}$ with $871 \mu \mathrm{m}$ radiation, and as such is very similar in form to $C R$ reflectivity signals seen from 2D electron gases in semiconductor heterostructures when the underlying reflectivity of the structure is high [18]. In contrast to the $-10 \%$ modulation of the reflectivity seen in the latter systems, however, the small skin depth of (BEDT-TTF) ${ }_{2} \mathrm{KHg}(\mathrm{SCN})_{4}$ means that the $\mathrm{CR}$ leads to only a $-0.6 \%$ change in reflectivity. At higher energies the dip becomes wider and shallower, whereas for longer wavelengths the CR is observed as a peak. The latter behavior is also seen in heterostructures under conditions of changing background reflectance [18]; the sharp change in behavior between 871 and 944 $\mu \mathrm{m}$ may be due to a low-energy lattice phonon. The cause of the broadening of the resonance at higher energies is as yet unknown; it may represent the onset of a resonant coupling to a phonon mode (cf. Ref. [18]). The weaker CR at higher fields (Fig. 2) also exhibits changes in shape as a function of wavelength (cf. GaAs heterostructure reflectance data in Ref. [18], Figs. 1, 3, and 4). The field positions of the $\mathrm{CR}$ features are plotted against energy in Fig. 3. Effective masses of $m_{\text {cycl }}^{*}=(0.40$ $\pm 0.02) m_{e}$ and $m_{\mathrm{cyc} 2}^{*}=(0.94 \pm 0.02) m_{e}$ are estimated from the linear slopes of the plots of radiation energy against $\mathrm{CR}$ field position.

We now consider the relation between the two effective masses $m_{\mathrm{cycl}}^{*}$ and $m_{\mathrm{cyc} 2}^{*}$ observed in CR and the effective masses $m_{\text {tr1 }}^{*}$ and $m_{\text {tr2 }}^{*}$ measured from the SdHO. A 


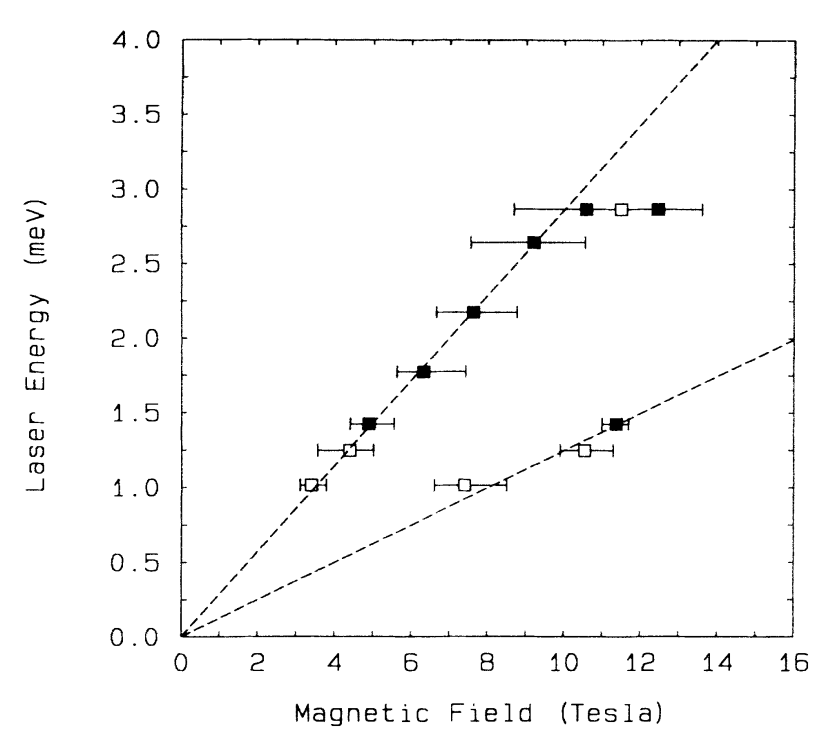

FIG. 3. CR positions at each energy. Solid squares correspond to reflectance minima and open squares to maxima. The horizontal bars correspond to the estimated widths of the CR features. The dashed and solid lines correspond respectively to fitted effective masses of $0.40 m_{e}$ and $0.94 m_{e}$.

closed Fermi surface is necessary for the observation of both $\mathrm{CR}$ and $\mathrm{SdHO}$. In addition, in a system such as (BEDT-TTF $)_{2} \mathrm{KHg}(\mathrm{SCN})_{4}$, one would expect the CR and SdHO to be similarly affected by the various scattering mechanisms present, i.e., there will be a correlation between the strength of the CR and the SdHO. We therefore assign the stronger $\mathrm{CR}$ to the 2D $V$-point hole pocket associated with the dominant 670-T SdHO and the weaker CR to the extra carrier pocket responsible for the secondary, higher-frequency SdHO series; however, note that, even if this assignment is inverted, the conclusions drawn below are unaffected.

Comparing data taken over comparable temperature ranges, the CR $(1.2 \mathrm{~K})$ yields effective masses of $0.40 m_{e}$ and $0.94 m_{e}$ while the SdHO $(0.5-4.0 \mathrm{~K})$ give values close to $2.0 m_{e}$ and $2.4 m_{e}$, respectively. We believe that this difference is a consequence of Kohn's theorem [8, 14], which states that at long wavelengths an external perturbation only couples to center-of-mass motion, which is independent of EEI. Thus CR, representing the excitation of a $q=0$ magnetoplasmon, measures the "bare" bandstructure mass, renormalized only by electron-phonon interactions [18], whereas the transport mass, representing the $q=\infty$ case of a magnetoplasmon [19], is also renormalized by EEI. Some violations of Kohn's theorem due to nonparabolicity have been observed in CR in highmobility semiconductor heterojunctions [19,20], but these tend to lead to a change in CR line shape, rather than a large shift in $\mathrm{CR}$ position. Thus a comparison between the measured $m_{\text {cyc }}^{*}$ and $m_{\mathrm{tr}}^{*}$ for (BEDT-TTF) ${ }_{2} \mathrm{KHg}-$ $(\mathrm{SCN})_{4}$ represents a direct gauge of the strength of the EEI in a $2 \mathrm{D}$ system as measured by the renormalization of the effective mass.

Effective masses may also be derived from the band structure calculated by Mori et al. [15]. On the basis of this band structure, we obtain a cyclotron mass of $1.2 m_{e}$ for the $V$-point hole pocket. The plasma frequencies are calculated to be $0.77 \mathrm{eV}$ for $\mathbf{E}$ polarized along $\mathbf{a}$, and 0.35 $\mathrm{eV}$ for $\mathbf{E}$ along c. Experimental plasma frequencies in this material have been measured by Tamura et al. [21] to be $1.1 \mathrm{eV}$ with the $E$ vector polarized along a, and 0.63 $\mathrm{eV}$ with the $E$ vector along c, suggesting that the true bandwidth is greater (and hence the effective mass is smaller) than predicted by Mori's band structure. Since the effective mass is inversely proportional to the square of the plasma frequency, we would expect a cyclotron mass of $0.47 m_{e}$ on the basis of the measured plasma frequencies. This value is similar to the measured effective mass of the stronger CR.

The optical mass measurements (from CR and plasma frequency) are both insensitive to $\mathrm{EEI}$ and indicate a larger bandwidth in (BEDT-TTF ${ }_{2} \mathrm{KHg}(\mathrm{SCN})_{4}$ than that predicted from band-structure calculations, which is in turn bigger than that deduced from transport measurements. A similar effect may take place in the $11-\mathrm{K}$ superconductor (BEDT-TTF $)_{2} \mathrm{Cu}(\mathrm{SCN})_{2}$, where the transport mass is -4 times larger than that expected from band-structure calculations [3], leading Toyota et al. [4] to point out that band-structure calculations in BEDTTTF salts almost always reproduce the experimentally observed Fermi-surface shapes fairly reliably, but overestimate the bandwidth (i.e., underestimate the effective mass). Toyota et al. showed that the observed narrow bandwidth must be due to a many-body renormalization, but in the absence of further data were unable to state whether this was due to electron-phonon interactions or EEI, or both. As electron-phonon interactions are expected to renormalize both $m_{\mathrm{cyc}}^{*}$ and $m_{\mathrm{tr}}^{*}$ equivalently [22], the experiments reported in this paper indicate that the major part of the observed difference between calculated and observed bandwidths is due to EEI, which lead to an enhancement of $\sim 5$ in the effective mass of the $V$ point holes.

The EEI responsible for the effective-mass renormalization can be described by a Hubbard model with an effective on-site Coulomb correlation energy $U_{\text {efr. }}$ Mass renormalization in the Hubbard model has been considered by Brinkman and Rice [23] using the Gutzwiller approximation. For a half-filled band the renormalization factor is $\left[1-\left(U_{\mathrm{eff}} / 8 \epsilon\right)^{2}\right]^{-1}$, where $\epsilon$ is the average energy of the electrons. For the present purposes a simplified view of the band structure of (BEDT$\mathrm{TTF})_{2} \mathrm{KHg}(\mathrm{SCN})_{4}$ [15] will be taken. A dimer is regarded as the basic unit, which produces two dimer bands, the upper band being half filled. The experimentally observed mass enhancements of order 2-5 imply values for $U_{\mathrm{eff}} / 8 \epsilon$ in the range $0.7-0.9$. The average energy of electrons in the upper subband is estimated to be $-60 \mathrm{meV}$ from the band structure [15]. This leads to a 
value for $U_{\mathrm{eff}}$ for the dimer in the range $0.4-0.5 \mathrm{eV}$; in this model $U_{\text {eff }}$ is associated with the intradimer correlation energy $V$ and the values obtained here are entirely consistent with typical values for $V$ derived from optical spectra of a wide range of charge-transfer salts [24].

In summary, we have measured $C R$ in (BEDTTTF $)_{2} \mathrm{KHg}(\mathrm{SCN})_{4}$ for the first time; the results indicate low carrier effective masses of $0.4 m_{e}$ and $0.94 m_{e}$. In contrast, transport measurements reveal much higher effective masses $\sim 2.0 m_{e}$ and $2.4 m_{e}$. We believe that the difference between the transport and cyclotron masses is an indication of the strength of EEI in the material.

This work is supported by the Science and Engineering Research Council in the United Kingdom, by Stichting voor Fundamenteel Onderzoek der Materie (FOM) in The Netherlands, and by the European Community Large Installations Plan. We would like to thank J. R. Cooper and R. H. Friend of Cambridge University for illuminating discussions, and K. van Hulst of the Nijmegen Magnet Laboratory for his support and encouragement.

[1] BEDT-TTF is bis(ethylenedithio)-tetrathiafulvalene.

[2] T. Ishiguro and K. Yamaji, in Organic Superconductors, edited by M. Cardona, P. Fulde, K. von Klitzing, and $\mathbf{H}$. J. Queisser, Springer Series in Solid-State Sciences Vol. 88 (Springer-Verlag, Berlin, 1990).

[3] F. L. Pratt et al., in The Physics and Chemistry of Organic Superconductors, edited by G. Saito and S. Kagoshima, Springer Proceedings in Physics Vol. 51 (Springer-Verlag, Berlin, 1990), p. 200.

[4] N. Toyota, E. W. Fenton, T. Sasaki, and M. Tachiki, Solid State Commun. 72, 859 (1989).

[5] M. Tokumoto et al., in Proceedings of the International Conference on Organic Superconductors, Lake Tahoe, 1990 (Plenum, New York, 1990).
[6] I. D. Parker et al., Synth. Met. 27, A387 (1988); A. G. Swanson et al., Solid State Commun. 73, 353 (1990); M. Tokumoto et al., Solid State Commun. 75, 439 (1990).

[7] A. S. Perel et al., Phys. Rev. Lett. 67, 2072 (1991).

[8] W. Kohn, Phys. Rev. 123, 1242 (1961).

[9] J. Larsen and C. Lenoir, Synthesis, 134 (1988).

[10] T. Sasaki, H. Sato, and N. Toyota, Synth. Met. 42, 2211 (1991).

[11] T. Sasaki et al., Solid State Commun. 75, 93 (1990); 75 , 97 (1990).

[12] M. Tokumoto et al., J. Phys. Soc. Jpn. 59, 2324 (1990).

[13] T. Osada et al., Synth. Met. 42, 2171 (1991); T. Osada et al., Phys. Rev. B 41, 5428 (1990).

[14] M. Doporto et al., in Proceedings of the Third International Symposium on Research in High Magnetic Fields, Amsterdam, 1991 [Physica (Amsterdam) B and C (to be published)]; F. L. Pratt et al., Phys. Rev. B (to be published).

[15] H. Mori et al., Bull. Chem. Soc. Jpn. 63, 2183 (1990).

[16] J. Singleton (unpublished).

[17] J. Wosnitza et al., Phys. Rev. Lett. 67, 263 (1991).

[18] C. J. G. M. Langerak et al., Phys. Scr. T39, 308 (1991).

[19] R. J. Nicholas et al., in High Magnetic Fields in Semiconductor Physics II, edited by G. Landwehr, Springer Series in Solid-State Sciences Vol. 87 (Springer-Verlag, Berlin, 1989), p. 449.

[20] M. Watts et al., in Proceedings of the International Conference on the Applications of High Magnetic Fields in Semiconductor Physics, Würzburg, 1990 (Springer, New York, to be published).

[21] M. Tamura et al., Synth. Met. 42, 2499 (1991).

[22] J. W. Wilkins, in Electrons at the Fermi Surface, edited by M. Springford (Cambridge Univ. Press, Cambridge, 1980), p. 46.

[23] W. F. Brinkman and T. M. Rice, Phys. Rev. B 2, 4302 (1970).

[24] S. Mazumdar and S. N. Dixit, Phys. Rev. B 34, 3683 (1986). 